\title{
The role of ultrasonography in assessing disease activity in patients with rheumatoid arthritis and associated fibromyalgia
}

\author{
Linda Jessica Ghib', Maria-Magdalena Tămaş', Laura Otilia Damian², Ioana Felea², \\ Laura Mirela Muntean', Nicolae Rednic ${ }^{3}$, Simona Rednic ${ }^{1}$
}

${ }^{1}$ Rheumatology Department, "Iuliu Haţieganu” University of Medicine and Pharmacy, ${ }^{2}$ Rheumatology Department, Clinical Emergency County Hospital, ${ }^{3} 4^{\text {th }}$ Medical Department, Railway Hospital, Cluj-Napoca, Romania

\begin{abstract}
Aim: The purpose of this study is to compare and correlate US evaluation with clinical scores of the disease activity in patients with rheumatoid arthritis (RA) and concomitant fibromyalgia (FM). Material and methods: Ten patients diagnosed with RA according to the 2010 ACR/EULAR classification criteria and associated FM based on the ACR 1990 classification criteria and two control groups, one with RA (10 patients) and one with FM (10 patients), were included. Clinical assessment was performed and the disease activity scores were calculated. Synovial/tenosynovial hypertrophy, fluid collections in grey scale (GS), and Power Doppler (PD) US assessed by US in the 28 joints included in the disease activity score 28 (DAS28). Results: GS US score and PD US scores were correlated with DAS28 only in patients with RA (Pearson r coefficients 0.3 and 0.5 ). Mean DAS28 score was significantly higher in the RA/FM group, compared to RA and FM (5.6 versus 4.6 versus 4.5, respectively). Patients with RA/FM had similar median US scores to RA patients, while in FM group significantly lower median US scores were detected (16 versus 9.5 versus 0 for GS US and 3.5 versus 1.5 versus 0 for PD US, respectively). Conclusions: Disease activity scores should be interpreted with caution in patients with RA and FM. When available, US should be used to guide treatment decisions in patients with RA and FM.
\end{abstract}

Keywords: rheumatoid arthritis, fibromyalgia, ultrasonography, DAS28

\section{Introduction}

Rheumatoid arthritis (RA) is a chronic disease characterized by synovial hypertrophy and joint destruction. In order to prevent destructive changes and maintain remission or low disease activity, a treatment algorithm based on the disease activity scores was recommended by an international task force [1]. The recommended disease activity scores are Disease Activity Score 28 (DAS28), Simplified Disease Activity Index (SDAI) and Clinical Disease Activity Index (CDAI) [2]. These scores include tender joint count (TJC) and swollen joint count

Received 10.06.2015 Accepted 05.07.2015

Med Ultrason

2015, Vol. 17, No 3, 339-344

Corresponding author: Linda Jessica Ghib

2-4 Clinicilor Street

400006 Cluj-Napoca, Romania

Phone $+40744-755586$

Fax +40264-431040

E-mail: ghib.linda@umfcluj.ro
(SJC), patient global assessment (PGA), physician global assessment (PhGA) and erythrocyte sedimentation rate (ESR) [2].

Fibromyalgia (FM), a chronic disease characterized by diffuse pain, sleep disturbances and somatic symptoms, is present in $17 \%$ of patients with RA [3]. RA patients with associated FM have higher disability index, lower quality of life, and higher medical costs [3]. It seems that an even higher percent of patients with RA may present features of FM and increased pain sensitivity without satisfying diagnostic criteria for FM. These patients have higher patient reported components of the disease activity scores [4]. Wolfe et al evaluated the effect of polysymptomatic distress, a composite measure of fibromyalgianess derived from the 2010 classification criteria for FM, on disease activity measurements in patients with RA [5]. Polysymptomatic distress influenced disease activity scores and authors concluded that these scores cannot discriminate between active disease and patient distress [5]. When DAS28 score was applied to 
patients with FM it did not differ significantly from the scores obtained for patients with RA. This raised the hypothesis that applying these scores in patients with RA and associated FM would cause an overestimation of disease activity [6]. In a prospective study, FM coexistence was an independent predictor of higher DAS28 score in patients with RA [7].

Ultrasonography (US) is an important tool for detecting synovitis, complementary to clinical examination [8]. Synovial hypertrophy and effusions are examined by using gray scale (GS) ultrasound and the neovascularisation within the synovitis is detected by using the Power Doppler (PD) US [8].

US has also been proven to predict RA radiological progression at the joint level and is a good marker of prognosis [9]. US scores have been developed and tested, with different number of joints examined and several scoring systems regarding GS and PD examination [10]. The most widely used scoring system for small joints and tenosynovitis is the one proposed by Backhaus et al [11]. The Sonography of Large Joints in Rheumatology (SOLAR) is the only validated score that evaluates semiquantitatively GS and PD in large joints [12].

The main aim of this study is to evaluate and to compare the US (in GS and PD) and DAS28 scores between RA, RA and associated FM and FM patients. Our secondary aim is to correlate the US scores with clinical disease activity in the aforementioned patient groups.

\section{Material and methods}

This cross-sectional case-control study was performed in the Rheumatology Clinic, Emergency Clinical County Hospital Cluj-Napoca between November 2014 and March 2015. Adult female patients who fulfilled $2010 \mathrm{ACR} / \mathrm{EULAR}$ classification criteria for RA [13] and 1990 classification criteria for FM [14] were consecutively enrolled from the outpatient clinic by their attending physicians (LOD, LMM, and IF). Two agematched control groups were included, one with RA and one with FM. Exclusion criteria consisted of any other concomitant inflammatory disease, infection, pregnancy, and lactation. The study was conducted according to the Declaration of Helsinki and the protocol was approved by "Iuliu Haţieganu" University of Medicine and Pharmacy Ethics Committee. All subjects signed a written informed consent.

Clinical scores for the disease activity and US scores were performed at 28 joints included in DAS28. Performance of US versus clinical assessment in patients with RA and concomitant FM and in the control groups was assessed.
US was performed by the same experienced ultrasonographer (MMT) ( $>5$ years experience). Both the clinician and the ultrasonographer were blinded to the patient's diagnosis.

\section{Clinical assessment}

All patients underwent evaluation of 28 joints: 1-5 metacarpophalangeal (MCP), 1-5 proximal interphalangeal (PIP), wrist, elbow, shoulder, knee bilaterally. TJC, SJC, PGA, PhGA, number of tender points for fibromyalgia (NTP) were recorded by one of the investigators (LJG). ESR was measured at 1 hour (normal $<20 \mathrm{~mm} / \mathrm{h}$ ). Additional data was retrieved from patients charts regarding duration of disease, IG M rheumatoid factor (RF) positivity (RF, normal $>32 \mathrm{UI} / \mathrm{ml})$ and anti-cyclic citrullinated peptide antibody positivity (aCCP, normal $>5$ $\mathrm{UI} / \mathrm{ml}$ ), current treatment. All patients completed health assessment questionnaire - disability index (HAQ-DI). DAS28 was calculated with ESR value. Values lower or equal to 2.6 were interpreted as remission, between 2.6 and 3.2 as low disease activity, between 3.2 and 5.1 as moderate disease activity, and $\geq 5.1$ as high disease activity. For CDAI remission was considered between $0-2.8$, low disease activity $2.9-10$, moderate disease activity 10.1-22 and high disease activity 22.1-76 [15,16].

\section{Ultrasound examination}

Ultrasound was performed by on Acuson S2000 (Siemens Healthcare), equipped with high frequency linear probes $(18 \mathrm{MHz})$. PD settings were optimized to low wall filters, a pulse repetition frequency of $900 \mathrm{~Hz}$ and the color gain was set below noise floor. Twenty eight joints included in DAS28 were examined. Synovial hypertrophy, tenosynovitis and fluid collections were assessed according to musculoskeletal ultrasound guidelines [11]. Wrists and MCP joints were examined in the longitudinal plane, from the dorsal aspect and PIP joints from the volar aspect to assess the presence of synovial fluid/ hypertrophy (GS) and synovial vascularisation (PD) (1 scan each joint). Compartment 6 of the dorsal wrist (extensor ulnari carpis tendon) and 1-5 finger flexor tendons were scanned for the detection of tenosynovitis (TS) in GS and PD US [17] in both hands. A total of 12 tendons were scanned for the detection of TS. Large joints were scanned according to the SOLAR score: shoulder - glenohumeral posterior transverse, axillary longitudinal (2 scans), elbow - humeroradial, humeroulnar, olecranon fossa (3 scans), knee - suprapatellar longitudinal, medial, and lateral femurotibial, dorsal longitudinal (4 scans) [12]. A total of 42 scans/ patient at joints level were obtained.

Semi-quantitative GS and PD scores (grade 0-3) were used for small joints [11], for large joints [12], and qualitative scores for GS TS (0-absent, 1- present) and 
semiquantitative (0-3) for PD TS [17]. A total score of $(0-3) \times 42$ for GS and for PD, respectively and 0-12 for GS TS and (0-3) x 12 for PD TS, was calculated for each patient.

\section{Statistical analysis}

Statistical analysis was performed with SPSS v20. Normal distributions were checked with histograms and Shapiro Wilk test. Data are represented as mean (SD) if normally distributed or median (IQR) for non normal distributions. Differences between groups were tested with One Way Anova for normally distributed data or Kruskal Wallis test for non-normally distributed data. Post hoc analysis was performed using Mann U Whitney test for each pair of groups with adjusted the $p$ value using the Bonferroni multiple comparison procedure $(\mathrm{p}<0.016)$. Testing for homogeneity of variance was performed with the Levene test. Correlations were calculated by using Pearson correlation coefficient. A significance value of $\mathrm{p}<0.05$ was used.

\section{Results}

Thirty female patients (10 with RA, 10 with RA and FM, and 10 with FM) were included and 840 joints were examined by US. Demographics, clinical data, and medication use in patients and disease controls are shown in Table I.

\section{Clinical assessment}

TJC and PGA were higher in the RA/FM group comparing to RA and FM, respectively. SJC and ESR were not significantly different between the RA and RA/FM group, but significantly lower in the FM group (Table II).

Table I. Demographic and disease characteristics of the study subjects.

\begin{tabular}{|c|c|c|c|c|}
\hline & $\operatorname{RA}(n=10)$ & RA with FM (n=10) & FM $(n=10)$ & $\mathbf{P}$ \\
\hline Age (mean, SD) & $57.9(14.8)$ & $58(7.1)$ & $49.2(10.6)$ & 0.37 \\
\hline \multicolumn{5}{|l|}{ Rheumatoid arthritis } \\
\hline Disease duration, months & $69(55,135)$ & $126(71.5,168)$ & - & 0.1 \\
\hline Rheumatoid factor positivity n (\%) & $8(80)$ & $5(50)$ & - & \\
\hline Anti CCP antibody positivity $n(\%)$ & $7(70)$ & $6(60)$ & - & \\
\hline DMARD n $(\%)$ & $10(100)$ & $10(100)$ & - & \\
\hline Biological DMARD n (\%) & $2(20)$ & $2(20)$ & & \\
\hline NSAIDS n (\%) & $3(30)$ & $7(70)$ & $8(80)$ & \\
\hline Prednisone n $(\%)$ & $2(20)$ & $2(20)$ & 0 & \\
\hline \multicolumn{5}{|l|}{ Fibromyalgia } \\
\hline Disease duration (months) & - & $42(16.2,61)$ & $44(3,85)$ & 0.5 \\
\hline Tender point count & $2(0,6)$ & $14.5(11.5,16.5)$ & $13.5(11,18)$ & 0.001 \\
\hline Antidepressants n (\%) & - & $2(20)$ & $4(40)$ & \\
\hline Anticonvulsants n ( \%) & - & $3(30)$ & $1(10)$ & \\
\hline
\end{tabular}

Values are expressed as mean (\%) or median (25,75 percentiles); CCP- cyclic citrullinated peptide; DMARD- disease modifying anti rheumatic drug; NSAID- non steroidal anti inflammatory drug; RA- rheumatoid arthritis; FM- fibromyalgia

Table II. Clinical and ultrasound characteristics in patients and disease controls

\begin{tabular}{lllll}
\hline & RA $(\mathbf{n}=\mathbf{2 8 0}$ joints) & RA with FM (n=280 joints) & FM (n=280 joints) & $\mathbf{p}$ \\
\hline TJC & $5.5(2,10)$ & $15(11.7,20.7)$ & $11.5(9,21.7)$ & $\mathrm{p}=0.07 *$ \\
SJC & $6(0.7,7.5)$ & $5(0.7,8)$ & $0(0,1.2)$ & $\mathrm{p}=0.08 * * *$ \\
PGH on VAS & $44.5(21)$ & $66(15)$ & $73(14.9)$ & $\mathrm{p}=0.04 *$ \\
PhGH & $39.5(17)$ & $32(14.7)$ & $14(14)$ & $\mathrm{p}=0.003^{* *}, * * *$ \\
ESR(mm/h) & $22(13.7,40)$ & $23(7.2,42.5)$ & $10(4.7,14.2)$ & $\mathrm{p}=0.02^{* * *}$ \\
DAS28 & $4.63(1.1)$ & $5.6(0.7)$ & $4.53(1.2)$ & $\mathrm{p}=0.03^{* *}$ \\
CDAI & $19.8(9.7)$ & $30.9(9.4)$ & $23.1(9)$ & $\mathrm{p}=0.05^{* *}$ \\
US GS score & $16(7.5,24.7)$ & $9.5(4.7,13)$ & $3(1.7,8.7)$ & $\mathrm{p}=0.05$ 而 \\
US PD score & $3.5(0,10.7)$ & $1.5(1,8)$ & $0(0,1)$ & $\mathrm{p}=0.008^{* * *}$ \\
HAQ & $0.83(0.7)$ & $1.56(0.4)$ & $1.07(0.6)$ & $\mathrm{p}=0.07$ \\
\hline
\end{tabular}

Values are expressed as mean (\%) or median (25,75 percentiles); *Differences significant for RA group;**Differences significant for RA/FM group;***Differences significant for FM group; $\uparrow$ No significant differences at post-hoc analysis; TJC- tender joint count, SJC- swollen joint count, PGH on VAS- patients global health on visual analogue scale (0-100), DAS28- disease activity score with 28 joints; CDAI- clinical disease activity; US GS score- ultrasonography gray scale score; US PD score- ultrasonography Power Doppler score; HAQ- health assessment questionnaire 


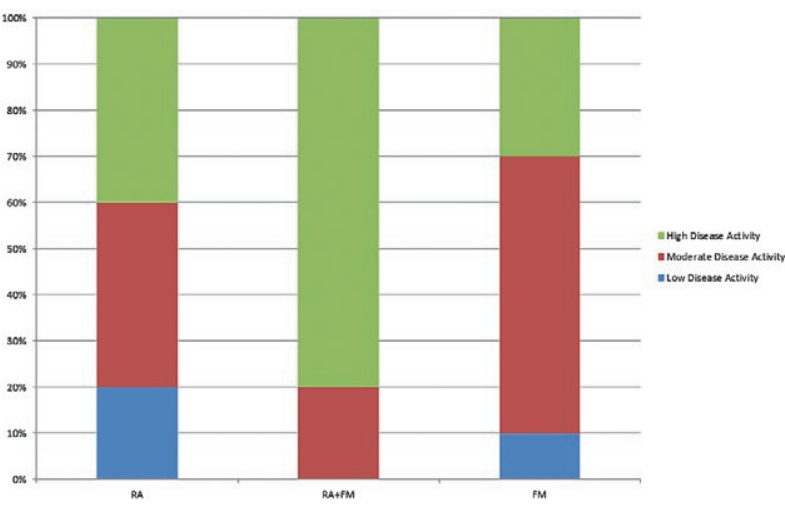

Fig 1. Disease activity categories according to DAS28 (ESR) scores. RA - rheumatoid arthritis; FM- fibromyalgia

Table III. Correlations between DAS28, CDAI, and US scores in patients and disease controls

\begin{tabular}{|c|c|c|c|c|}
\hline & \multicolumn{2}{|c|}{ DAS28 } & \multicolumn{2}{|c|}{ CDAI } \\
\hline & $\mathrm{r}$ & $\mathrm{p}$ & $r$ & $\mathrm{p}$ \\
\hline \multicolumn{5}{|l|}{ RA } \\
\hline GS US & 0.3 & 0.04 & 0.5 & 0.03 \\
\hline PD US & 0.5 & 0.05 & 0.6 & 0.02 \\
\hline \multicolumn{5}{|l|}{$\mathbf{R A}+\mathbf{F M}$} \\
\hline GS US & 0.1 & 0.3 & 0.3 & 0.5 \\
\hline PD US & 0.5 & 0.2 & 0.5 & 0.2 \\
\hline \multicolumn{5}{|l|}{ FM } \\
\hline GS US & 0.1 & 0.7 & -0.7 & 0.01 \\
\hline PD US & -0.1 & 0.7 & -0.4 & 0.03 \\
\hline
\end{tabular}

$\mathrm{r}$ - Pearson correlation coefficient, GS US - gray scale ultrasonography score; PD US - power Doppler ultrasonography score; RA - rheumatoid arthritis; FM - fibromyalgia; DAS28: disease activity score in 28 joints; CDAI - clinical disease activity index

Patients with RA/FM had higher DAS28 scores, eight out of ten patients being classified as having high disease activity. By contrast, only 4 out of 10 RA presented DAS28 scores corresponding to high disease activity (fig 1).

\section{Ultrasound scores}

GS US score ranged from 0 to 36 in the RA group, from 0 to 24 in the RA/FM group, and from 0 to 11 in the FM group. PD scores varied from 0 to 24 in the RA group, from 0 to 11 in the RA/FM group and from 0 to 2 in the FM group. Median GS and PD US scores were significantly different between the 3 groups (Table II). At post hoc analysis significant differences were found between the FM and RA groups for PD US ( $p=0.014)$, but not for GS US $(p=0.04)$. The same results were obtained when we compared median US scores between the RA / FM group and FM group: $p=0.06$ for GS US and $p=0.003$ for PD US. No significant differences were noted between US scores in the RA and RA/FM groups ( $p=0.2$ for GS US and $\mathrm{p}=0.6$ for PD US).
US scores correlated moderately only with DAS28 and CDAI in patients with RA, and not in patients with RA/FM. These scores were inversely correlated with DAS28 and CDAI in FM patients (Table III).

TS GS scores had minimum and maximum values from 0 to 6 for the RA group, 0 to 4 in the RA/FM group, and 0 to 2 in the FM group. TS PD scores ranged between minimum and maximum values of 0 to 6 for RA, 0 to 2 for RA/FM, and 0 to 1 for FM.

\section{Discussions}

Our results showed that DAS28 is not a reliable measure of disease activity in patients with RA and associated FM, due to the higher TJC and PGH. US examination can assess more objectively the disease activity by evaluating the presence of the joint synovitis and tenosynovitis, especially in the presence of PD signal in these patients.

These findings are similar to those of previous studies. Ranzolin et al found that patients with RA and associated FM had higher disease activity scores, disability, and worse quality of life compared to those with RA alone [7]. Another study found that US synovitis score in 7 joints (US 7) was a better tool of assessing disease activity than composite disease activity scores in patients with RA and associated FM. The PD US7 score correlated with DAS28 only in patients without FM [17]. Our findings show small correlation coefficients between GS US and DAS28 and moderate for PD US and DAS28, suggesting that PD US could perform better in assessing disease activity in patients with RA and associated FM.

In our study, although ESR and SJC values were similar between the RA and RA/FM group, DAS 28 (ESR) and CDAI values were significantly higher in the RA/FM group, pointing out as previously found in other studies $[6,18]$, to higher TJC and PGA in patients with associated FM. TJC was significantly lower in patients with RA compared to those with RA/ FM and FM probably due to central sensitivity mechanisms of pain and not to inflammation [3].

PD US was demonstrated to be a sensitive method for assessment of the clinical activity in RA patients [19] and PD activity was comparable to that of magnetic resonance imaging [20]. In our study, PD US scores were similar between the RA and RA/FM group, although DAS 28 scores were higher in the RA/FM group. GS scores were significantly different between the 3 groups of patients, but pair wise comparisons did not reach levels of statistical significance, probably due to the small number of subjects.

Another core measurement in RA is the HAQ-DI [21]. RA patients have a higher disability index that the 
normal population [22] and patients with RA/FM exhibit higher HAQ scores than those with FM [7]. In our study, patients with RA/FM had the highest HAQ score, but values did not reach a statistical significance. Longer RA duration in the RA/FM group could also explain the higher disability index in this group.

When employing treat to target or tight control treatment regimens for RA, FM status should be assessed and decisions should be taken carefully, considering objective individual components of DAS28 such as SJC or ESR/ CRP. Another option would be to take treatment decisions based on US findings.

In many countries reimbursement of biological treatment and monitoring during treatment is based on DAS28 scores [23]. Patients should not be labeled as non responders to biological therapy only based on the DAS28 score, but also based on US examination if available. A recent study by Kay et al found that a significant proportion of patients in the United States RA registry have clinically active disease despite normal values of ESR and CRP [24]. Another study found that ESR and CRP are weakly correlated between each other and with disease activity in RA [25]. Thus, acute phase reactants like ESR and CRP cannot be used to distinguish between active and inactive disease in all patients with RA and associated FM.

To our knowledge this is the first study that directly compared 28 joints ultrasound score of patients with RA, RA/FM and FM.

One of the limitations of our study is the small number of patients included. We did not use the SDAI because CRP was not available for all patients. In a recent review by Gaujoux-Viala et al the DAS 28, CDAI, and SDAI were shown to perform similarly concerning sensitivity to change, intraobserver reproductibility, concordance, construct validity and performance [26]. We chose to use dorsal scans for MCP joints based on findings of Szkudlarek et al that showed a higher percent of dorsal compared to palmar US involvement in RA [27]. Other studies showed a preponderance of palmar involvement in MCP joints of patients with RA [28]. Both sides should probably be scanned in order not to miss involved joints and we acknowledge this as another limitation of our study.

Recent studies suggest that central mechanisms of pain play an important role in rheumatoid arthritis and patients exhibit features of fibromyalgia [4,5]. In this context US assessment of disease activity in the presence of low pain thresholds should be studied.

\section{Conclusions}

Patients with RA and associated FM have similar US scores with RA patients, but higher disease activity scores due to increased TJC and reported PGA. Only PD US significantly differ in patients with RA versus FM. DAS 28 was correlated with PD US only in RA and not in RA/FM or FM patients. In order to assess disease activity objectively disease activity scores should be interpreted with caution and when available US should be used to guide treatment decisions in patients with RA and FM.

Acknowledgement: This paper was published under the framework of the European Social Fund, Human Resources Development Operational Programme 20072013, project no. POSDRU/159/1.5/S/138776

\section{Conflict of interest: none}

\section{References}

1. Smolen JS, Aletaha D, Bijlsma JW, et al. Treating rheumatoid arthritis to target: recommendations of an international task force. Ann Rheum Dis 2010; 69: 631-637.

2. Anderson J, Caplan L, Yazdany J, et al. Rheumatoid arthritis disease activity measures: American College of Rheumatology recommendations for use in clinical practice. Arthritis Care Res (Hoboken) 2012; 64: 640-647.

3. Wolfe F, Michaud K. Severe rheumatoid arthritis (RA), worse outcomes, comorbid illness, and sociodemographic disadvantage characterize ra patients with fibromyalgia. $\mathrm{J}$ Rheumatol 2004; 31: 695-700.

4. Joharatnam N, McWilliams DF, Wilson D, Wheeler M, Pande I, Walsh DA. A cross-sectional study of pain sensitivity, disease-activity assessment, mental health, and fibromyalgia status in rheumatoid arthritis. Arthritis Res Ther 2015; 17: 11.

5. Wolfe F, Michaud K, Busch RE, et al. Polysymptomatic distress in patients with rheumatoid arthritis: understanding disproportionate response and its spectrum. Arthritis Care Res (Hoboken) 2014; 66: 1465-1471.

6. Leeb BF, Andel I, Sautner J, Nothnagl T, Rintelen B. The DAS28 in rheumatoid arthritis and fibromyalgia patients. Rheumatology 2004; 43: 1504-1507.

7. Ranzolin A, Brenol JC, Bredemeier M, et al. Association of concomitant fibromyalgia with worse disease activity score in 28 joints, health assessment questionnaire, and short form 36 scores in patients with rheumatoid arthritis. Arthritis Care Res 2009; 61: 794-800.

8. Naredo E, Bonilla G, Gamero F, Uson J, Carmona L, Laffon A. Assessment of inflammatory activity in rheumatoid arthritis: a comparative study of clinical evaluation with grey scale and power Doppler ultrasonography. Ann Rheum Dis 2005; 64: 375-381.

9. Colebatch AN, Edwards CJ, Østergaard M, et al. EULAR recommendations for the use of imaging of the joints in the clinical management of rheumatoid arthritis. Ann Rheum Dis 2013; 72: 804-814. 
10. Chakr RMDS, Mendonça JA, Brenol CV, Xavier RM, Brenol JC. Assessing rheumatoid arthritis disease activity with ultrasound. Clin Rheumatol 2013; 32: 1249-1254.

11. Backhaus M, Burmester GR, Gerber T, et al. Guidelines for musculoskeletal ultrasound in rheumatology. Ann Rheum Dis 2001; 60: 641-649.

12. Hartung W, Kellner H, Strunk J, et al. Development and evaluation of a novel ultrasound score for large joints in rheumatoid arthritis: one year of experience in daily clinical practice. Arthritis Care Res (Hoboken) 2012; 64: 675-682.

13. Aletaha D, Neogi T, Silman AJ, et al. 2010 Rheumatoid arthritis classification criteria: An American College of Rheumatology/European League Against Rheumatism collaborative initiative. Arthritis Rheum 2010; 62: 2569-2581.

14. Wolfe F, Smythe HA, Yunus MB, Bennett RM, Bombardier C, Goldenberg DL, et al. The American College of Rheumatology 1990 Criteria for the classification of fibromyalgia. Report of the Multicenter Criteria Committee. Arthritis Rheum 1990; 33: 160-172.

15. Prevoo ML, Van't Hof MA, Kuper HH, Van Leeuwen MA, Van de Putte LB, Van Riel PL. Modified disease activity scores that include twenty-eight-joint counts development and validation in a prospective longitudinal study of patients with rheumatoid arthritis. Arthritis Rheum 1995; 38: 44-48.

16. Aletaha D, Nell VP, Stamm T, et al. Acute phase reactants add little to composite disease activity indices for rheumatoid arthritis: validation of a clinical activity score. Arthritis Res Ther 2005; 7: R796-R806.

17. Backhaus TM, Ohrndorf S, Kellner H, et al. The US7 score is sensitive to change in a large cohort of patients with rheumatoid arthritis over 12 months of therapy. Ann Rheum Dis 2013; 72: 1163-1169.

18. Ton E, Bakker MF, Verstappen SM, et al. Look beyond the disease activity score of 28 joints (DAS28): tender points influence the DAS28 in patients with rheumatoid arthritis. J Rheumatol 2012; 39: 22-27.

19. Naredo E, Bonilla G, Gamero F, Uson J, Carmona L, Laffon A. Assessment of inflammatory activity in rheumatoid arthritis: a comparative study of clinical evaluation with grey scale and power Doppler ultrasonography. Ann Rheum Dis 2005; 64: 375-381.
20. Terslev L, Torp-Pedersen S, Savnik A, et al. Doppler ultrasound and magnetic resonance imaging of synovial inflammation of the hand in rheumatoid arthritis: A comparative study. Arthritis Rheum 2003; 48: 2434-2441.

21. Felson DT, Anderson JJ, Boers M, et al. The American College of Rheumatology preliminary core set of disease activity measures for rheumatoid arthritis clinical trials. The Committee on Outcome Measures in Rheumatoid Arthritis Clinical Trials. Arthritis Rheum 1993; 36: 729-740.

22. Sokka T, Krishnan E, Häkkinen A, Hannonen P. Functional disability in rheumatoid arthritis patients compared with a community population in Finland. Arthritis Rheum 2003; 48: 59-63.

23. Orlewska E, Ancuta I, Anic B, et al. Access to biologic treatment for rheumatoid arthritis in Central and Eastern European (CEE) countries. Med Sci Monit 2011; 17: SR1SR13.

24. Kay J, Morgacheva O, Messing SP, et al. Clinical disease activity and acute phase reactant levels are discordant among patients with active rheumatoid arthritis: acute phase reactant levels contribute separately to predicting outcome at one year. Arthritis Res Ther 2014; 16: R40.

25. Keenan RT, Swearingen CJ, Yazici Y. Erythrocyte sedimentation rate and C-reactive protein levels are poorly correlated with clinical measures of disease activity in rheumatoid arthritis, systemic lupus erythematosus and osteoarthritis patients. Clin Exp Rheumatol 2008; 26: 814-819.

26. Gaujoux-Viala C, Mouterde G, Baillet A, et al. Evaluating disease activity in rheumatoid arthritis: Which composite index is best? A systematic literature analysis of studies comparing the psychometric properties of the DAS, DAS28, SDAI and CDAI. Joint Bone Spine 2012; 79: 149155.

27. Szkudlarek M, Klarlund M, Narvestad E, et al. Ultrasonography of the metacarpophalangeal and proximal interphalangeal joints in rheumatoid arthritis: a comparison with magnetic resonance imaging, conventional radiography and clinical examination. Arthritis Res Ther 2006; 8: R52.

28. Scheel AK, Hermann KG, Kahler E, et al. A novel ultrasonographic synovitis scoring system suitable for analyzing finger joint inflammation in rheumatoid arthritis. Arthritis Rheum 2005; 52: 733-743. 